\title{
Three-Dimensional Numerical Simulation of Rear Point Contact Crystalline Silicon Solar Cells
}

\author{
M.Zanuccoli ${ }^{1}$, H.-W. Guo ${ }^{2}$, E. Sangiorgi ${ }^{1}$, C. Fiegna ${ }^{1}$ \\ ${ }^{1}$ ARCES-DEIS, University of Bologna and IUNET, Via Venezia 52, 47521, Cesena, Italy \\ Phone number:+0039 0547 339539, e-mail: mazanuccoli@arces.unibo.it \\ ${ }^{2}$ APPLIED MATERIALS, Inc., 3050 Bowers Avenue, Santa Clara, CA 95054-3299 U.S.A.
}

\begin{abstract}
High efficiency monocrystalline solar cells commonly adopts rear point contacts of limited extension and passivation of the uncontacted bottom silicon surface region in order to improve performance. Modeling and analysis of advanced solar cells is strategic to optimize the device design and to minimize the losses. Several competing physical mechanisms must be accounted for in order to properly analyze rear point contact solar cells and a three-dimensional (3D) analysis of complex geometries is required. In this work we describe the issues related to the design of the mesh grid for the 3D numerical simulations, the definition of appropriate boundary conditions and the main adopted assumptions. Examples of numerical simulations are reported.
\end{abstract}

\section{Keywords}

Rear point contact solar cells, three-dimensional numerical simulation, advanced solar cells.

\section{Introduction}

The recombination losses occurring at the rear contact of crystalline solar cell significantly limit the conversion efficiency. Conventional solar cells, which feature a base contact metalization extended over the whole back silicon surface, are affected by significant recombination losses at the metal-semiconductor interface. Highefficiency silicon solar cells usually feature local point contacts at the back surface, allowing the passivation of the uncontacted back silicon surface region (see Fig. 1). This back-contact scheme is commonly adopted by PERC (passivated emitter and rear cell) and PERL (passivated emitter, rear locally diffused) solar cells and enables the fabrication of single-junction cells featuring record conversion efficiency well above $20 \%$ [1], [2].

As discussed in [3], the optimum design of rear point contact solar cells requires a trade-off between several effects such as the reduction of the surface recombination velocity at the passivated back interface, the larger bottom effective internal reflectivity of passivated surface compared to metal-contacted region and the enhancement of series parasitic resistances that is mainly due to the back contact-resistance and to the base spreading resistance associated to 3D conduction paths occurring when the extension of the contacted region is much smaller than the cell area.
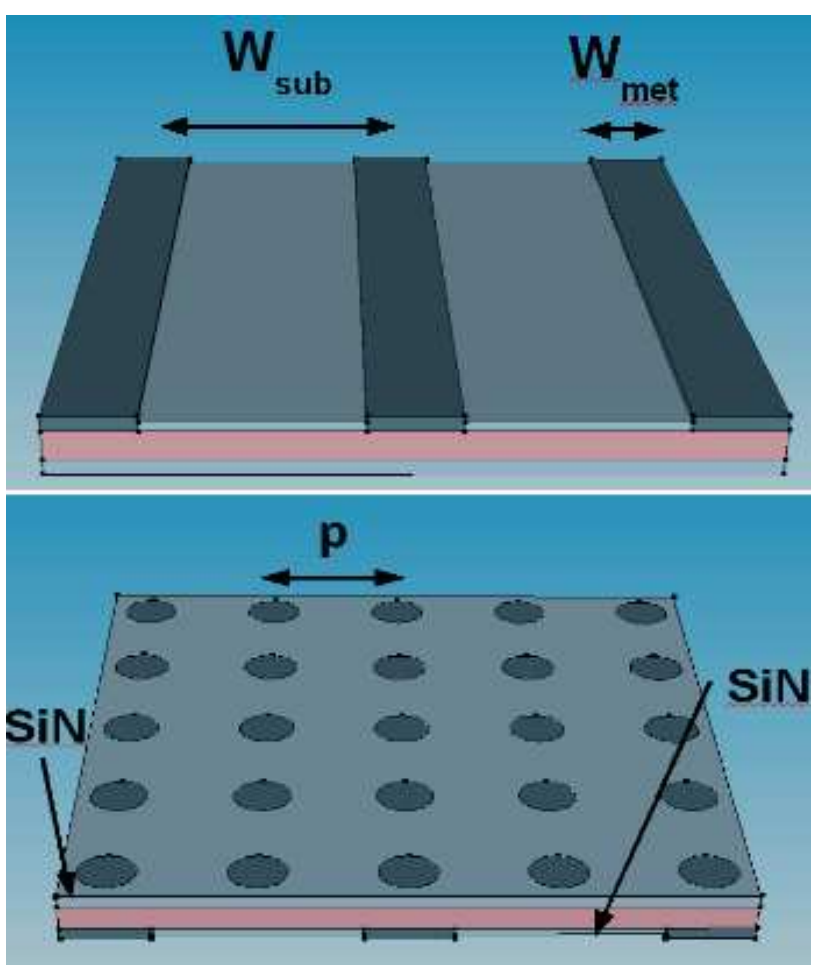

Fig. 1 Sketch of a rear point contact solar cell. On the top side, $\mathrm{W}_{\text {sub }}$ denotes the front contact pitch and $\mathrm{W}_{\text {met }}$ the front contact finger width. The figure on the bottom highlights the rear side of the device featuring the holes equally distributed with period $p$. The interface that is not contacted by the metallization is passivated by silicon nitride $(\mathrm{SiN})$ or silicon oxide.

Typical rear point contact solar cells feature circular contacts uniformly distributed on the bottom surface with diameter $s$ and repetition period (pitch) $p$ (see Fig. 1). In the following we denote by $f$ the metallization fraction at the rear side, defined as the ratio of the contacted area to the total cell area and expressed by Equation (1):

$$
f=\pi \cdot\left(\frac{s}{2 p}\right)^{2}
$$

The non-metalized rear surface is typically passivated by using silicon nitride or silicon dioxide in order to reduce the density of surface defects and suppress recombination [3]. Given the complex 3D geometry, modeling of rear point contact solar cells is fundamental in order to optimize the device design.

Previous works were limited to two-dimensional numerical simulation approaches [4] or relied on quasi- 
3D approaches based on 1D simulation coupled to semiempirical analytical models for the calculation of the spreading resistance and of an effective back surface recombination velocity $\left(\mathrm{BSRV}_{\text {eff }}\right)$ as function of the rear surface contact geometry, the surface recombination velocities at passivated interface, the surface recombination velocity at metal-silicon interface and the electric properties of the base such as its resistivity [5].

Since rear point contact solar cells feature a threedimensional pattern, it is necessary to perform a truly three-dimensional (3D) simulation to properly model physical mechanisms like the current crowding occurring close to the contacted regions that cannot be fully appreciated by limiting the analysis to simple twodimensional domains. On the other hand, 3D analysis requires a large mesh grid for numerical discretization and very long computation time on high-performance workstations to carry out the simulation task.

In this work the 3D analysis of of rear point contact cells is performed by using Sentaurus Sdevice by Synopsys [6] coupled to an in-house developed optical simulator based on the propagation of electromagnetic waves for the calculation of the optical generation rate map inside the semiconductor.

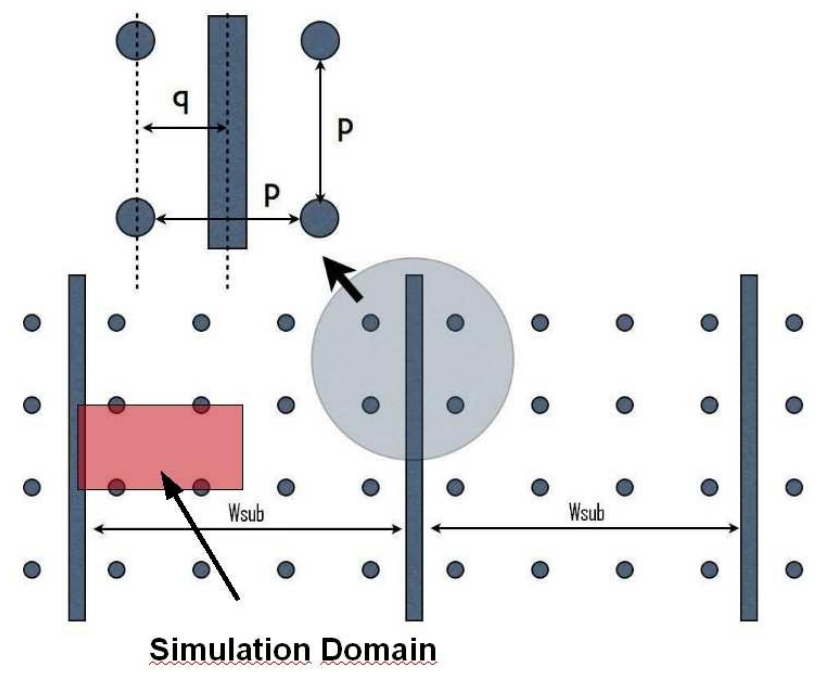

Fig. 2. Top-down two-dimensional representation of a rear point contact solar cell. The simulation domain is highlighted in red. In our simulations the front contact fingers are aligned to the holes of the rear surfaces $(q=0)$. The front contact and the hole pitches are denoted by $W_{s u b}$ and $p$, respectively. In this figure $W_{s u b}>p$.

\section{Simulated device and modeling approach}

The simulated device is a monocrystalline solar cell with a $65 \Omega /$ sq homogeneous emitter and a rear point contact geometry (Fig. 1). The front contact pitch $W_{\text {sub }}$ and width $W_{\text {met }}$ are set to $2000 \mu \mathrm{m}$ and $100 \mu \mathrm{m}$, respectively. Both front and rear uncontacted surfaces are passivated by $70 \mathrm{~nm}$ thick silicon nitride layers. The wafer thickness is $180 \mu \mathrm{m}$ while the resistivity of the p-type base is $0.5 \Omega \mathrm{cm}$ as suggested in [3] in order to avoid the rectifying action of the $\mathrm{Al} / \mathrm{p}-\mathrm{Si}$ interfaces.
The physical models adopted for the numerical simulations include the high-field and doping dependent mobility model as well the Schenk Band Gap narrowing [7]. Fermi statistics is adopted in order to correctly deal with high-doping concentration regions in the emitter. Minority carrier lifetime in the silicon bulk and surface recombinations velocities at passivated interfaces and contacted interfaces have been set so as to investigate the dependence of the cell efficiency on the material quality and process conditions.

The influence of the metallization fraction on the main figures of merit such as the short circuit current density (Jsc), the open circuit voltage (Voc), the Fill-Factor (FF) and cell efficiency, is analyzed by varying the contact size at constant pitch.

In order to minimize the requirements in terms of memory and processing time, proper simulation domains and mesh grids should be carefully chosen. Under the assumptions that the front contact pitch $\mathrm{W}_{\text {sub }}$ is larger than the rear contact pitch $p$ and that the ratio of the front contact pitch to the hole pitch is an integer number, the 3D simulation domain is selected so as to exploit the symmetry of the device (Fig. 2); the length and width of the domain are set to half of the front contact pitch and to the hole pitch of the rear surface, respectively, while the height of the domain equals the wafer thickness.

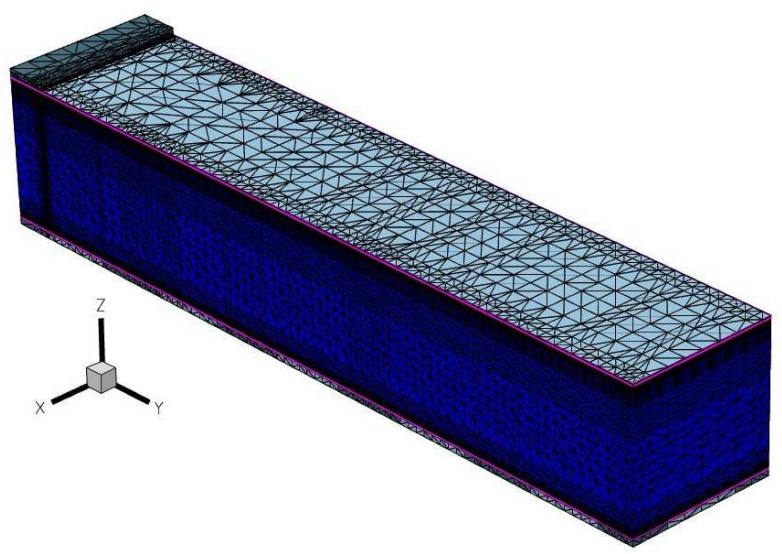

Fig. 3. Example of 3D mesh for numerical simulation. The mesh grid is particularly refined in the emitter region and in the portions of the device close to the holes and the front contact fingers. In this example the hole pitch $p$ and the front contact pitch $W_{s u b}$ are $200 \mu \mathrm{m}$ and $2000 \mu \mathrm{m}$, respectively.

Concerning the discretization mesh, a refined grid step is required in the emitter region in the vicinity of the junction, where carrier/doping gradients and the electric field intensity are large. The mesh refinement is progressively reduced while moving away from the junction, inside the base region. The grid step along $x$ and $y$ (Fig. 3) must be properly set in the vicinity of the rear contacts where 3D currents spreading occurs. The typical number of grid nodes adopted in our simulations is 700.000. Fig. 3 reports an example of 3D mesh grid for a rear point contact solar cell with $\mathrm{p}=200 \mu \mathrm{m}$ and $\mathrm{s}=100 \mu \mathrm{m}$.

The series contact resistance associated to the front contact fingers is calculated according to [8] by assuming the finger's length, height and width equal to $3 \mathrm{~cm}, 20 \mu \mathrm{m}$ 
and $100 \mu \mathrm{m}$, respectively. The sheet resistivity of the metal and the contact resistivity for the front fingers are set to $\rho_{\mathrm{M}}=6 \times 10^{-6} \Omega \mathrm{cm}$ and $\rho_{\mathrm{c}}=2 * 10^{-3} \Omega \mathrm{cm}^{2}$, respectively. Concerning the back contact, the only parasitic series resistance accounted for is that of the bottom metallization, for which the contact resistivity is set to $\rho_{\mathrm{c}}=2 * 10^{-3} \Omega \mathrm{cm}^{2}$.

Fig. 4 summarizes the electrical boundary conditions adopted in the simulations.

\section{Optical simulation}

Optical generation rate profiles are calculated on the basis of a simulation of plane-waves propagation in silicon assuming direct illumination with a standard AM1.5G spectrum $\left(1000 \mathrm{~W} / \mathrm{m}^{2}\right)$ with wavelength within the range $300 \mathrm{~nm}-1200 \mathrm{~nm}$. The surrounding medium is air.

At the top surface we use a combination of measured optical spectral reflectivity for textured surfaces and analytical Lambertian light trapping theory in order to calculate the carrier generation map which is then imported in the Synopsys device simulator. The multiple bounces of light inside the device are described analytically in terms of a geometric progression of plane waves.

The shadowing caused by front fingers is assumed ideal and the internal reflectance is the same for subsequent light bounces. Since we perform a 1D optical simulation, the internal bottom reflection coefficient $R_{b i}$ is assumed uniform at the rear interface and it is weighted on the metallization fraction according to:

$$
R_{b i}=\left(R_{b i, m}-0.25 f\right)
$$

where $R_{b i, m}$ is the internal bottom reflection coefficient of the silicon-dielectric interface and its value set to 0.9 [9]. According to equation (3), $\mathrm{R}_{\mathrm{bi}}$ equals 0.65 for a fully contacted back surface $(\mathrm{f}=1.0)$.

The adopted optical boundary conditions are summarized in Fig. 4.

\section{Simulation Results}

We simulated the rear point contact solar cell by assuming a minority carrier lifetime in the bulk equal to $200 \mu \mathrm{sec}$, a front surface recombination velocity of 1000 $\mathrm{cm} / \mathrm{sec}$ and BSRV $=200 \mathrm{~cm} / \mathrm{s}$ at passivated interfaces. The recombination velocity for electrons and holes at the metal-semiconductor interface is set to $10^{5} \mathrm{~cm} / \mathrm{s}$.

The calculated dependence of the output parameters on the metallization fraction are reported in Fig. 5 for the case $p=500 \mu \mathrm{m}$, neglecting the effects of series resistance in order to emphasize the influence of the base spreading resistance, the internal bottom internal reflectance and the effective bottom surface recombination. The reported values for short circuit current density, open circuit voltage, fill-factor and efficiency are normalized to those of the conventional cell $(f=1.0)$.

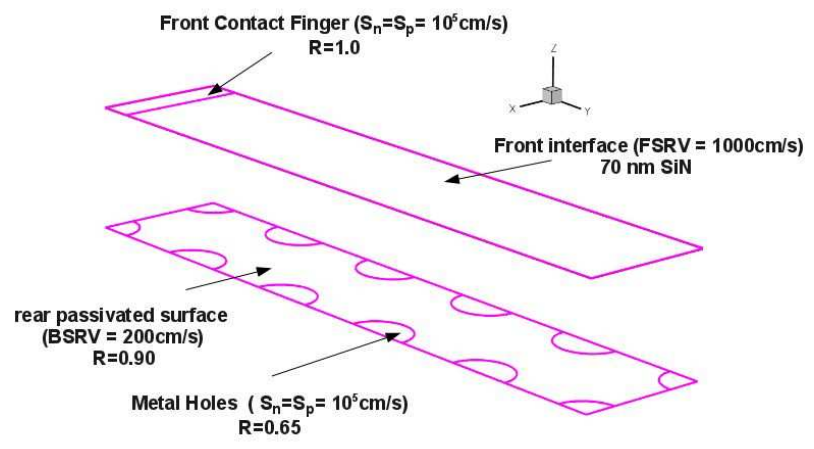

Fig. 4. Electrical and optical boundary conditions adopted for the simulations. The internal bottom reflection coefficient is function of the metalization fraction. The front contact finger is assumed ideal in terms of reflectivity coefficient and shadowing. On the side interfaces of the device the ideal Neumann boundary condition is assumed. At electrodes the recombination velocity is set to $10^{5} \mathrm{~cm} / \mathrm{s}$ for both electrons $\left(\mathrm{S}_{\mathrm{n}}\right)$ and holes $\left(\mathrm{S}_{\mathrm{p}}\right)$.

Both Voc and Jsc feature a monotonic trend as a function of the metallization fraction $f$; this trend is due to the reduction of the effective surface recombination velocity at the rear interface and the increase of the effective internal bottom reflectance as the metallization fraction decreases. As consequence of the reduction of the metallization fraction, the base series spreading resistance increases and this leads to a degradation of the fill-factor. An optimum value $f_{0}$ of the metallization fraction can be found and its value is within the range $1 \%-3 \%$ as shown in Fig. 6, where the effect of the contact resistance is included.

Table I. - Summary of results with the hole pitch $p$ as parameter. The minimum hole size is $25 \mu \mathrm{m}$.

\begin{tabular}{|c|c|c|c|c|c|c|}
\hline$p$ & $s$ & $f_{o}$ & Jsc & Voc & FF & Eff \\
\hline$[\mu \mathrm{m}]$ & {$[\mu \mathrm{m}]$} & {$[\%]$} & & & & \\
\hline & & 100 & 1.000 & 1.000 & 1.000 & 1.000 \\
\hline 200 & 25 & 1.23 & 1.080 & 1.035 & 0.995 & 1.113 \\
\hline 500 & 75 & 1.77 & 1.085 & 1.040 & 0.996 & 1.124 \\
\hline 1000 & 200 & 3.08 & 1.087 & 1.040 & 0.997 & 1.126 \\
\hline
\end{tabular}

The base spreading resistance -as reported in [5]depends both on the rear contact size and pitch; this translates into a weak dependence of the efficiency on the contact pitch (Fig. 6). Table 1 shows the results from simulations with the rear contact pitch $p$ as parameter assuming as minimum hole size $s=25 \mu \mathrm{m}$; for each considered value of the pitch $(p=200 \mu \mathrm{m}, p=500 \mu \mathrm{m}$ and $1000 \mu \mathrm{m}$ ), the optimum value of metallization fraction $\mathrm{f}_{0}$ is reported with the associated values of the main figures of merit. The largest enhancement of efficiency relative to the conventional cell predicted by simulations is about $13 \%$ for the case $p=1000 \mu \mathrm{m}, s=200 \mu \mathrm{m}, f=3.14 \%$; for the optimum configuration the Fill-Factor is lower (0.997) than that of the reference cell (normalized to 1.00) but both Jsc and Voc are larger (the normalized values are 1.087 and 1.040, respectively), leading to larger conversion efficiency. 

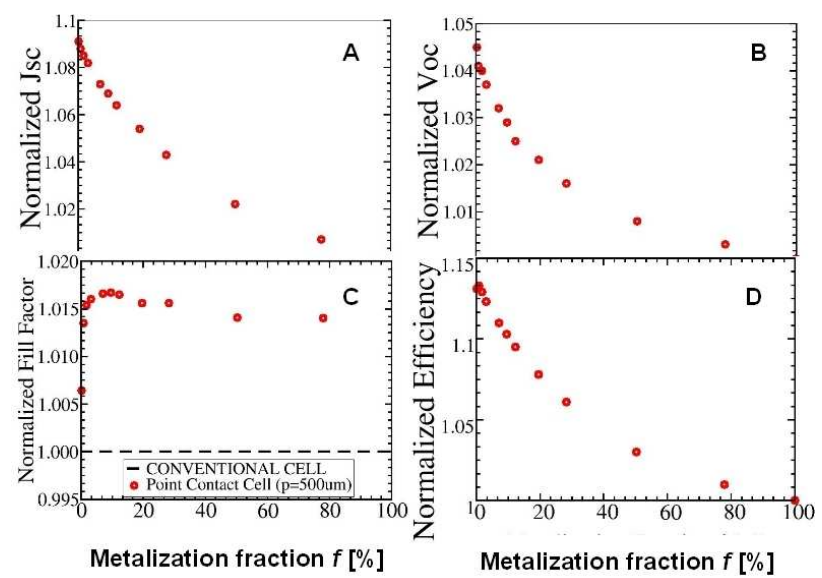

Fig. 5. Simulation of rear point contact cells with $p=500 \mu \mathrm{m}$, $W_{s u b}=2000 \mu \mathrm{m}$. From the upper left side: (A) short circuit current $\mathrm{J}_{\mathrm{sc}}$, (B) open circuit voltage $\mathrm{V}_{\mathrm{oc}}$, (C) Fill-Factor FF and (D) Efficiency versus the metallization fraction $f$. Results are normalized to the values calculated for the conventional cell $(\mathrm{f}=1.0)$. Parasitic contact series resistances are not included.

The current crowding effects occurring at the back contacts are illustrated in Fig. 7, that reports the total current map at $0 \mathrm{~V}$ bias (short circuit) under illumination by a monochrome wave $(1200 \mathrm{~nm})$.

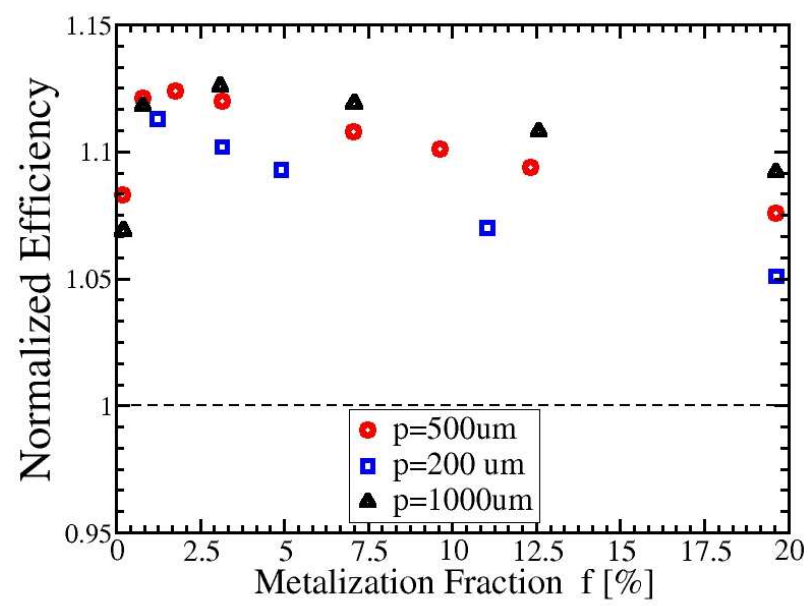

Fig. 6. Results from simulation of the rear point contact cells with $W_{\text {sub }}=2000 \mu \mathrm{m}$. The hole pitch $p$ is a parameter. All results are normalized to the conventional cell and the contact series resistance is taken into account.

\section{Conclusion}

An approach to model and optimize the design of the base of high performance monocrystalline silicon solar cell is presented. In particular the dependence of the conversion efficiency on the rear contact geometry is investigated for rear point contact solar cells. Numerical simulations have been performed by assuming a 3D simulation domain showing that the optimum metallization fraction value, which depends on a trade-off between several competing mechanisms like the surface recombination, the internal reflection of the light at the bottom interface and the series parasitic spreading resistance, is within the range $1 \%-3 \%$.

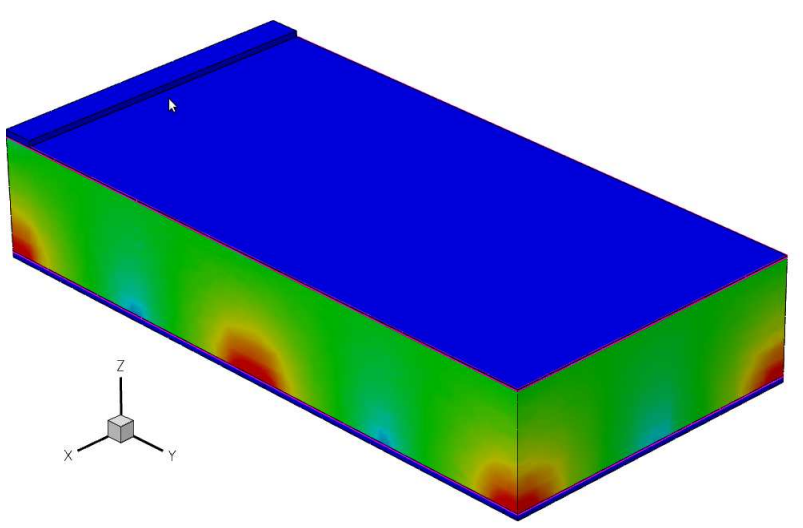

Fig. 7. Total current map at $0 \mathrm{~V}$ bias conditions for a monochrome wave $(1200 \mathrm{~nm})$. The point contact geometry is defined by $p=500 \mu \mathrm{m}, s=75 \mu \mathrm{m}$.

The enhancement of efficiency with respect to the conventional cell predicted by our simulations is about 13\%. The developed simulation procedure will allow further activities including an accurate modeling of the rear passivated interfaces, the analysis of the influence of several electrical parameters like the back surface recombination velocity, the substrate resistivity, the bulk minority lifetime and a detailed analysis of the main loss mechanisms.

\section{References}

[1] J. Zhao, A. Wang, M.A. Green, "24\% efficient PERL structure silicon solar cells", Photovoltaic Specialists Conference, 1990., Conference Record of the Twenty First IEEE

[2] Z. Shi, S. Wenham and J. Ji, "Mass Production of The Innovative Pluto Solar Cell Technology", Photovoltaic Specialists Conference (PVSC), 2009 34th IEEE

[3] A. G. Aberle, "Crystalline Silicon Solar Cells, Advanced Surface Passivation and Analysis", Centre for Photovoltaics Engineering, University of New South Wales, 2004, Australia

[4] A.G. Aberle, G. Heiser and M.A. Green, "Twodimensional Numerical Optimization Study of The Rear Contact Geometry of High-efficiency Silicon Solar Cells", J.Appl.Phys. 75 (10), 1994

[5] H. Plagwitz, M. Schaper, J. Schmidt, B. Terheiden and R. Brendel, "Analytical Model For The Optimization of Locally Contacted Solar Cells", Photovoltaic Specialists Conference, 2005.

[6] "Sentaurus Device User Guide, Version C-2009.06, June 2009", Synopsys

[7] P.P. Altermatt, A.Schenk, F. Geelhaar, G. Heiser, "Reassessment of the intrinsic carrier density in crystalline silicon in view of band-gap narrowing", Journal of Applied Physics, Vol. 93, N. 3

[8] A.Goetzberger, J.Knobloch, B. Vo $\beta$, “Crystalline Silicon Solar Cells", John Wiley \& Sons, 1998

[9] A. Upadhyaya, V. Yelundur, S. Ramanathan, J-H. Lai, V. Upadhyaya, A. Rohatgi, I.Koehler, "Enhanced Front and Rear dielectric passivation for commercially grown Czochralski silicon for high efficiency solar cells", 34th IEEE Photovoltaic Specialists Conference (PVSC), 2009 